\title{
Chest Computed Tomography Abnormalities and Their Relationship to the Clinical Manifestation of Respiratory Syncytial Virus Infection in a Genetically Confirmed Outbreak
}

\author{
Daijiro Nabeya ${ }^{1}$, Takeshi Kinjo ${ }^{1}$, Gretchen Lynn Parrott ${ }^{1}$, Sawako Nakachi ${ }^{2}$, \\ Tomoko Yamashiro ${ }^{1}$, Nanae Ikemiyagi ${ }^{1}$, Wakako Arakaki ${ }^{1}$, Hiroaki Masuzaki ${ }^{2}$ and Jiro Fujita ${ }^{1}$
}

\begin{abstract}
:
Studies reporting chest images of respiratory syncytial virus (RSV)-induced lower respiratory tract infection (LRTI) in an outbreak setting and their relationship to the clinical manifestation are limited. During a genetically confirmed RSV outbreak, eight patients underwent both chest X-ray and computed tomography (CT). Among these, 5 cases had newly appearing abnormalities on CT, although chest X-ray was able to detect abnormalities in only 2 cases (40\%). Although bronchial wall thickening was common, other findings and their distribution were variable, even in an outbreak setting. All patients with both a history of anticancer chemotherapy against hematological cancer and lower respiratory symptoms, such as wheezing, sputum, and hypoxemia, had abnormalities on $\mathrm{CT}$, suggesting that these two factors might be important for predicting the existence of LRTI in RSV-infected patients.
\end{abstract}

Key words: respiratory syncytial virus, outbreak, lower respiratory tract infection, bronchial wall thickenings, chest $\mathrm{CT}$

(Intern Med 59: 247-252, 2020)

(DOI: 10.2169/internalmedicine.3117-19)

\section{Introduction}

Respiratory syncytial virus (RSV) is well known as a virus causing lower respiratory tract infection (LRTI) in infants and immunocompromised adults (1-3). Bronchopneumonia patterns are common findings of RSV-induced LRTI (RSV-LRTI); however, RSV-LRTI often shows variable findings on chest imaging (4-11). Although some factors, such as the virulence of RSV and host immune status, might affect the chest computed tomography (CT) findings of RSVLRTI $(12,13)$, the detailed mechanisms remain unknown.

From this perspective, an RSV outbreak due to genetically identical RSV is an ideal setting for investigating the chest images of RSV-LRTI. Thus far, however, there has only been one report showing the chest images of RSVLRTI in the outbreak setting (11). In addition, reports inves- tigating the correlation between the clinical manifestations and abnormal findings on chest CT remain rare (14).

In 2014, a genetically identical RSV outbreak occurred in the hemato-oncology ward of the University of the Ryukyus Hospital (15), and a subset of RSV-infected patients underwent both chest X-ray and CT during the outbreak. We herein report our analysis of the radiological findings and the clinical manifestations in the outbreak.

\section{Case Report}

\section{Patients}

During the 2014 outbreak, RSV infection was diagnosed by reverse transcriptase polymerase chain reaction using respiratory specimens, and genetic proximity was confirmed by a phylogenetical analysis (15). Eight RSV-infected patients

${ }^{1}$ Department of Infectious, Respiratory, and Digestive Medicine, Graduate School of Medicine, University of the Ryukyus, Japan and ${ }^{2}$ Division of Endocrinology, Diabetes and Metabolism, Hematology and Rheumatology, Graduate School of Medicine, University of the Ryukyus, Japan Received for publication March 31, 2019; Accepted for publication August 1, 2019

Correspondence to Dr. Takeshi Kinjo, t_kinjo@med.u-ryukyu.ac.jp 


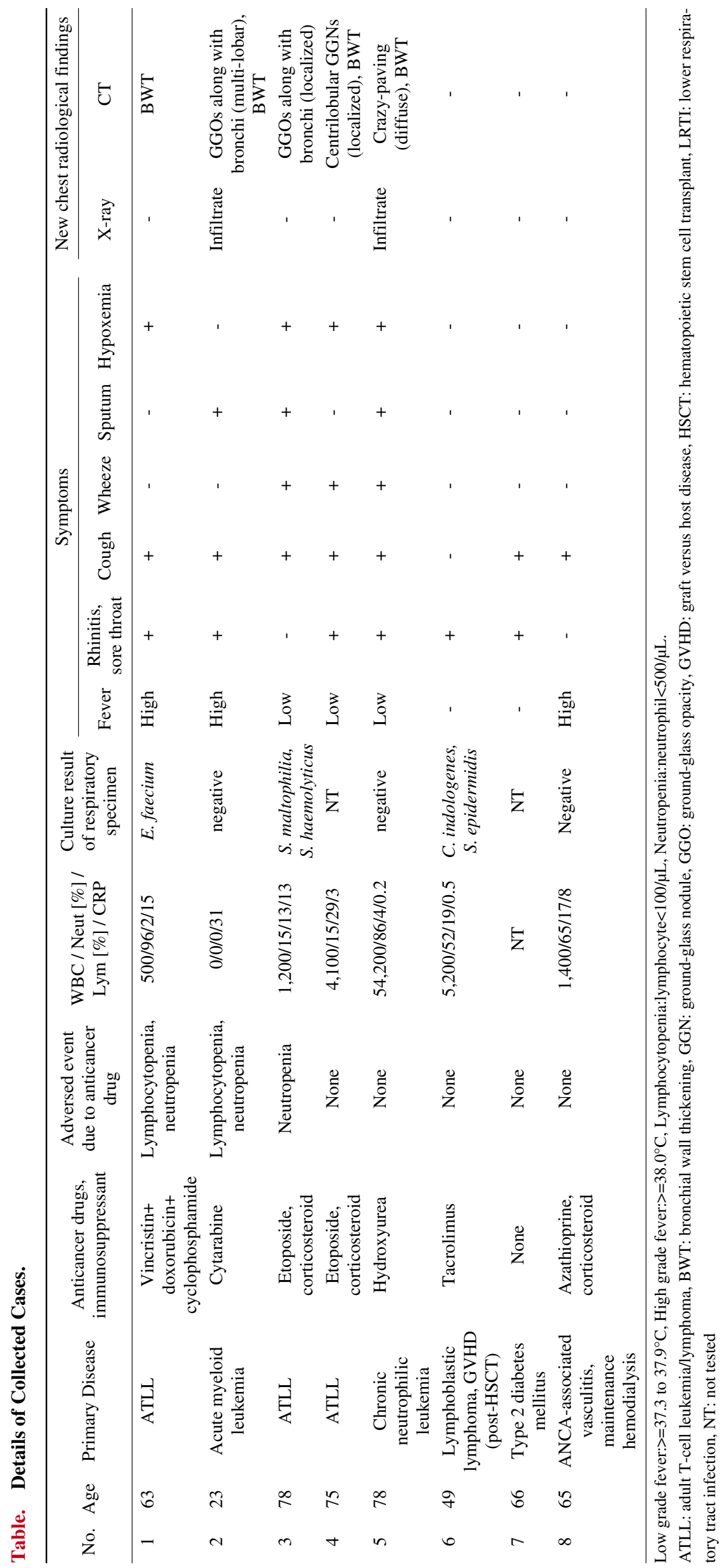




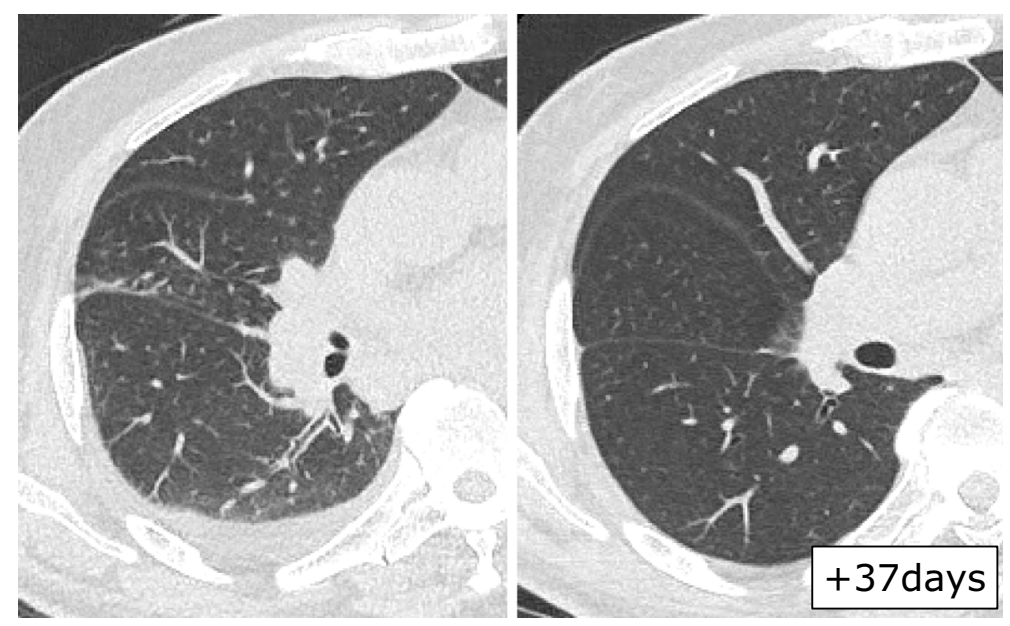

Figure 1. Chest CT findings in case 1. The first CT examination (left) was performed 10 days after the supposed onset date. Bronchial wall thickening in the lower right lobe was observed. Thirty-seven days later, these findings had disappeared (right).

underwent both chest X-ray and CT during the outbreak. The clinical information and chest $\mathrm{X}$-ray findings were retrospectively reviewed from patient medical records within one week of the chest CT scan.

\section{Ethics}

The medical records and radiological images of the collected cases were retrospectively reviewed, with identifying information removed. This study was reviewed and approved by the Clinical Research Ethics Committee of University of the Ryukyus.

\section{Results}

The patients' background characteristics, clinical manifestations, laboratory data, and findings from chest imaging are shown in Table. Seven cases (88\%) received various immunosuppressive treatment, and none had airway diseases, such as bronchial asthma. Five cases (63\%) had newly appearing abnormal findings on chest CT; however, no abnormal findings were detected by chest X-ray in 3 of those 5 patients $(60 \%)$. Most cases had upper respiratory symptoms, including rhinitis and sore throat. However, lower respiratory symptoms, such as wheeze, sputum, and hypoxemia, were only seen in patients with newly appearing findings on chest CT. Bacterial culture for respiratory specimens was performed in six cases, and bacteria were detected in three cases; however, no patient was diagnosed with bacterial pneumonia or treated with targeted antibiotics against these detected bacteria. In addition, no cases received antiviral therapy against RSV.

Chest CT findings for the five patients are shown in Figs. 1-5. The most common finding among these patients was bronchial wall thickenings; seen in 4 of 5 patients $(80 \%)$. In terms of the findings in the parenchymal lesion, the patterns and distributions were varied and inconsistent. Two cases showed ground-glass opacities (GGOs) along the bronchi; however, the distribution differed, as one was a localized GGO and the other a multilobar GGO. One case showed ground-glass nodules with a tree-in-bud pattern. The remaining case showed diffuse GGOs with centrilobular septal thickening, also known as the "crazy-paving" pattern. Three cases underwent two or more CT scans during the course of their disease, and the abnormal findings on chest CT disappeared (Fig. 1, 2, 5).

\section{Discussion}

In the present study, all patients who had lower respiratory symptoms, such as wheezing, sputum, and hypoxemia, had abnormal findings on chest CT. Indeed, sputum and hypoxemia are included as criteria for LRTI disease according to the guideline for respiratory virus infection in leukemia patients (16). In addition, all cases receiving chemotherapy for hematological malignancy also had abnormal findings on chest CT. This is probably associated with bone marrow suppression by cytotoxic anticancer drugs. Of note, lymphocytopenia and neutropenia are considered as risk factors for LRTI in RSV-infected cases (16). Therefore, it might be advised to perform chest $\mathrm{CT}$ in order to examine the existence of LRTI for RSV-infected hematological malignancy patients with both a history of anticancer chemotherapy and lower respiratory symptoms.

In RSV-LRTI, CT is more sensitive for detecting pulmonary findings than chest X-ray (6), as a bronchopneumonia pattern is more common in RSV-LRTI than an alveolar pattern $(5,7,11)$, and chest X-ray rarely detects the findings of a bronchopneumonia pattern. The present study showed that a bronchopneumonia pattern was indeed the most common (case 2 and 3), and chest X-ray missed these abnormal findings in most cases. Therefore, chest CT, rather than X-ray, should be performed to confirm LRTI in RSV-infected patients with lower respiratory symptoms. However, chest Xray is appropriate for evaluating pulmonary overexpansion 


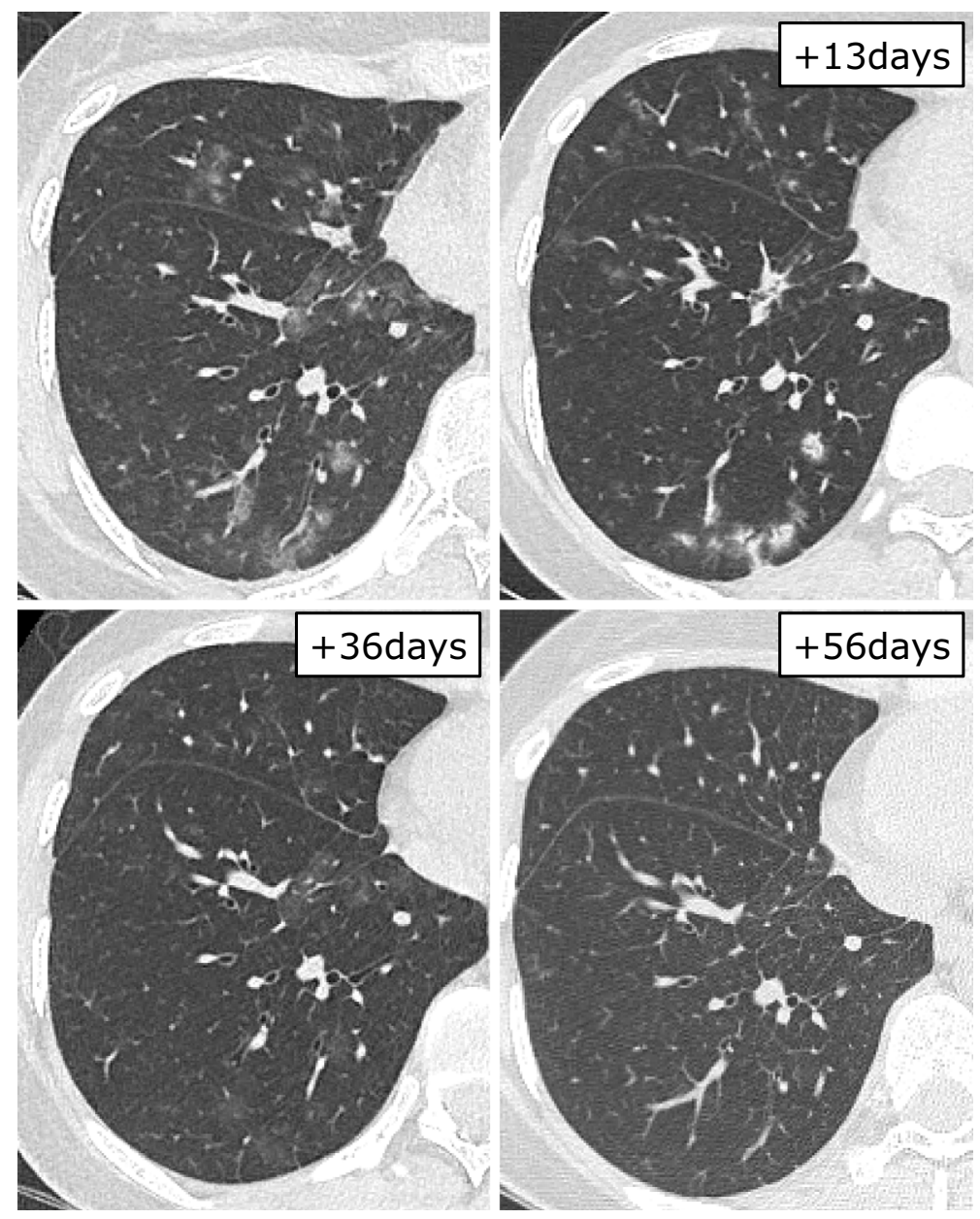

Figure 2. Chest CT findings in case 2. The first CT examination (upper left) was performed seven days after the supposed onset date. Multi-lobar ground-glass opacities along bronchi are shown. Bronchial wall thickening was observed in the upper right lobe. These lesions became patchy infiltrate and then gradually disappeared.
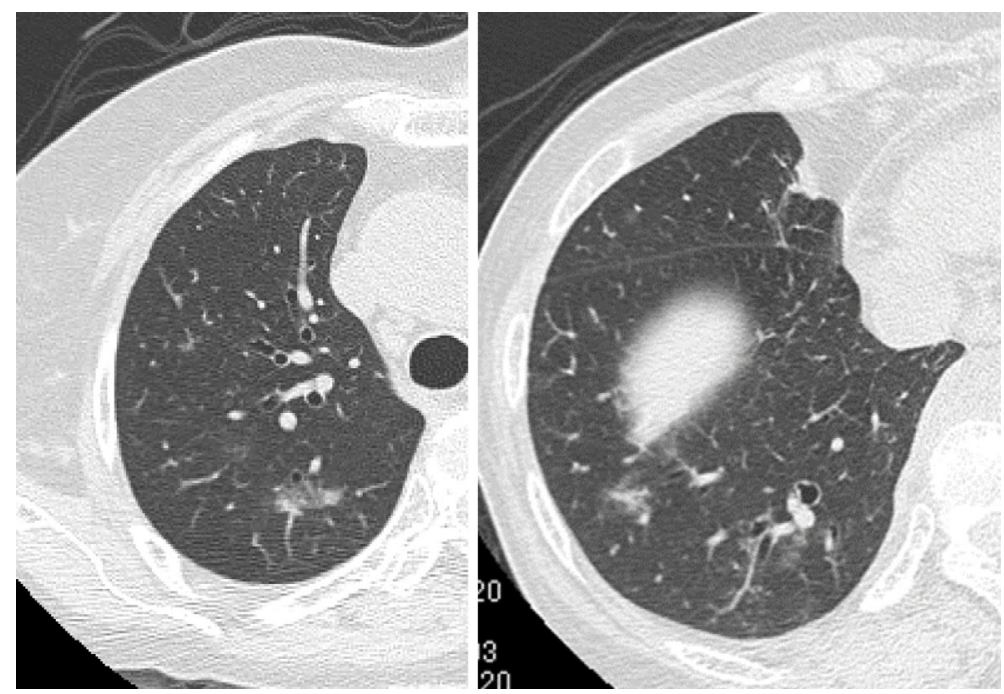

Figure 3. Chest CT findings in case 3. The CT examination was performed four days after the supposed onset date. Multi-lobar ground-glass opacities along bronchi were detected.

due to bronchiolitis in infants (17). Although bronchiolitis caused by RSV is relatively common in infants (18), it is not common in adults $(11,19)$. In fact, overexpansion due to bronchiolitis was not observed in any of our eight cases.

This study shows no trends for abnormal chest findings, even in the outbreak setting, which is consistent with a pre- 
vious report by Mayer et al. (11). Several reasons may explain this. First, although the pathophysiology remains unknown, the host's immunological status might affect CT findings. Immune responses by lymphocytes and neutrophils are correlated with disease severity, which may affect the patterns of abnormal CT findings (13). Indeed, the patients in our study had different primary diseases and received varying immunosuppressive therapies, as shown in the Table. Second, bacterial co-infection might affect the radiological findings. However, bronchioalveolar lavage fluid was collected from only one patient, and most specimens for bacterial culture were sputum; therefore, the possibility of contamination by oral bacteria could not be excluded. In addition, the bacteria detected in our study were uncommon pathogens for causing pneumonia. Indeed, no patient was

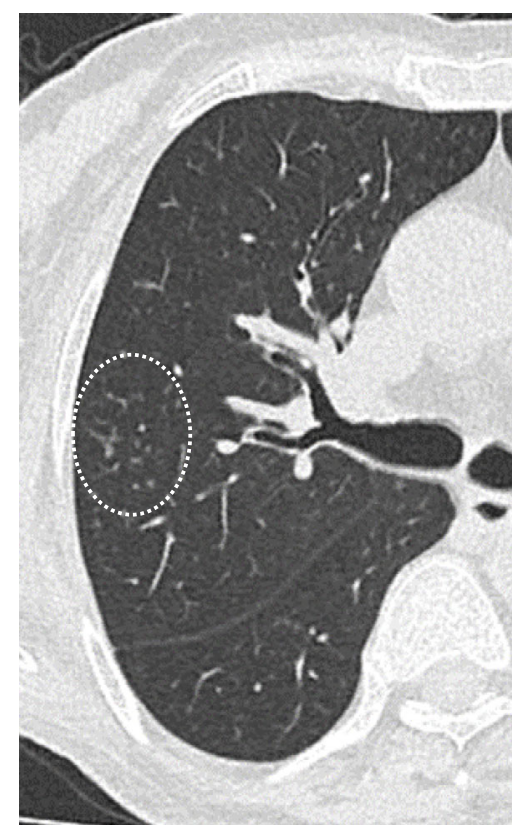

Figure 4. Chest CT findings in case 4. The CT examination was performed nine days after the supposed onset date. Localized ground-glass nodules were mainly seen in the centrilobular area (dotted line circle). Bronchial wall thickening was also seen. treated with antibiotics targeting these detected bacteria. Although the influence of these bacteria on CT findings is unknown, it is possible that these pathogens were colonized, rather than co-infection, so their impact on $\mathrm{CT}$ might be limited.

Although various findings are seen in patients with RSVLRTI (9), many reports have shown that nodules and tree-inbud patterns are relatively common $(5-8,10)$. Kim et al. compared the CT findings among RSV, parainfluenza virus, and influenza virus, demonstrating the frequent observation of bronchial wall thickening while consolidation and GGOs were rarely seen in RSV-LRTI compared to the other two viruses (4). Miller et al. reported that an airway-centric pattern, consisting of tree-in-bud opacities and bronchial wall thickening, was characteristic of RSV-LRTI compared to other respiratory viruses (9). It has also been reported that nodules and tree-in-bud patterns were seen during the early phase of RSV pneumonia $(9,11)$. Thus, although the findings and distributions for chest CT in cases 2, 3, and 4 varied, they were categorized as airway-centric patterns, and differences may exist due to the progression of disease. Case 4, which had tree-in-bud nodular opacities, progressed to respiratory failure after the CT scan, suggesting that these findings had been observed in the early phase of RSV pneumonia. Case 5 was initially diagnosed with idiopathic pulmonary alveolar hemorrhaging due to the hemoptysis and crazy-paving pattern on chest CT. The crazy-paving pattern is uncommon for RSV-LRTI; however, a previous case report showed that RSV infection can present as a crazypaving pattern in patients receiving hematopoietic stem cell transplantation (20).

\section{Conclusions}

Chest CT showed variable findings for RSV infection, even during an outbreak setting. In most cases, chest X-ray failed to detect abnormalities. In RSV-infected hematological malignancy patients receiving anticancer chemotherapy, lower respiratory symptoms may predict the existence of CT abnormalities due to RSV-LRTI.
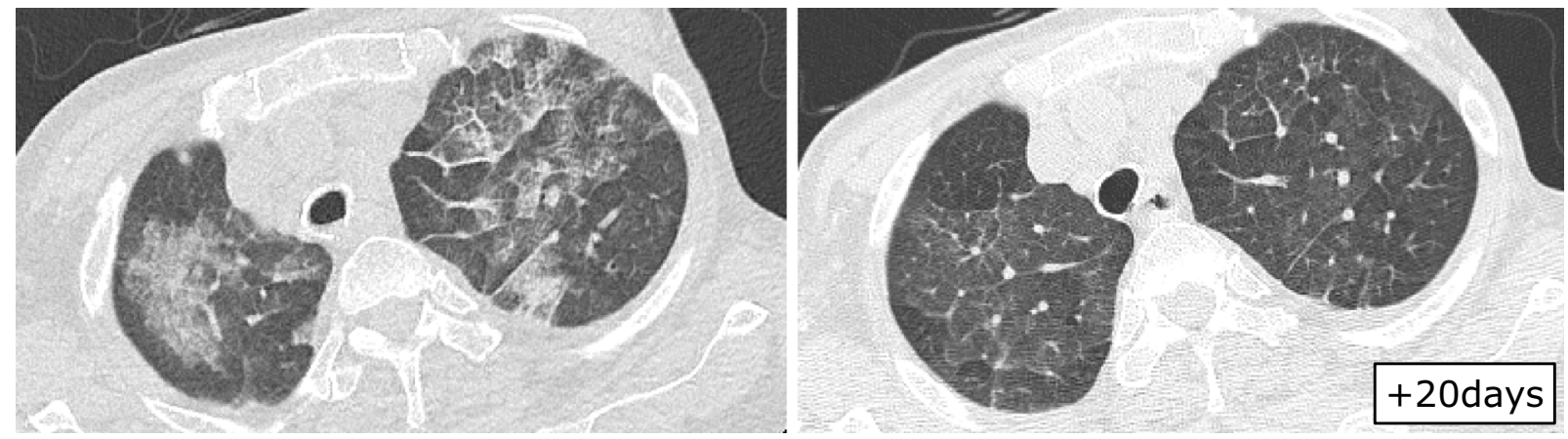

Figure 5. Chest CT findings in case 5. The first CT (left) was performed one day after the supposed onset date. A diffuse crazy-paving pattern, centrilobular septal thickening, and ground-glass opacities were seen. Twenty day later, these findings had become sparse (right). 
The authors state that they have no Conflict of Interest (COI).

\section{References}

1. Pilie P, Werbel WA, Riddell J, Shu X, Schaubel D, Gregg KS Adult patients with respiratory syncytial virus infection: impact of solid organ and hematopoietic stem cell transplantation on outcomes. Transpl Infect Dis 17: 551-557, 2015.

2. Kim YJ, Guthrie KA, Waghmare A, et al. Respiratory syncytial virus in hematopoietic cell transplant recipients: factors determining progression to lower respiratory tract disease. J Infect Dis 209: 1195-1204, 2014.

3. Lehners N, Schnitzler P, Geis S, et al. Risk factors and containment of respiratory syncytial virus outbreak in a hematology and transplant unit. Bone Marrow Transplant 48: 1548-1553, 2013.

4. Kim MC, Kim MY, Lee HJ, et al. CT findings in viral lower respiratory tract infections caused by parainfluenza virus, influenza virus and respiratory syncytial virus. Medicine (Baltimore) 95 e4003, 2016.

5. Ko JP, Shepard JA, Sproule MW, et al. CT manifestations of respiratory syncytial virus infection in lung transplant recipients. J Comput Assist Tomogr 24: 235-241, 2000.

6. Ariza-Heredia EJ, Fishman JE, Cleary T, Smith L, Razonable RR, Abbo L. Clinical and radiological features of respiratory syncytial virus in solid organ transplant recipients: a single-center experience. Transpl Infect Dis 14: 64-71, 2012.

7. Gasparetto EL, Escuissato DL, Marchiori E, Ono S, Frare e Silva RL, Müller NL. High-resolution CT findings of respiratory syncytial virus pneumonia after bone marrow transplantation. AJR Am J Roentgenol 182: 1133-1137, 2004.

8. Escuissato DL, Gasparetto EL, Marchiori E, et al. Pulmonary infections after bone marrow transplantation: high-resolution CT findings in 111 patients. AJR Am J Roentgenol 185: 608-615, 2005.

9. Miller WT, Mickus TJ, Barbosa E, Mullin C, Van Deerlin VM, Shiley KT. CT of viral lower respiratory tract infections in adults: comparison among viral organisms and between viral and bacterial infections. AJR Am J Roentgenol 197: 1088-1095, 2011.

10. Syha R, Beck R, Hetzel J, et al. Human metapneumovirus (HMPV) associated pulmonary infections in immunocompromised adults--initial CT findings, disease course and comparison to respiratory-syncytial-virus (RSV) induced pulmonary infections.
Eur J Radiol 81: 4173-4178, 2012.

11. Mayer JL, Lehners N, Egerer G, Kauczor HU, Heußel CP. CTmorphological characterization of respiratory syncytial virus (RSV) pneumonia in immune-compromised adults. Rofo 186: 686692, 2014.

12. Midulla F, Nenna R, Scagnolari C, et al. How respiratory syncytial virus genotypes influence the clinical course in infants hospitalized for bronchiolitis. J Infect Dis 219: 526-534, 2019.

13. Russell CD, Unger SA, Walton M, Schwarze J. The human immune response to respiratory syncytial virus infection. Clin Microbiol Rev 30: 481-502, 2017.

14. Shiley KT, Van Deerlin VM, Miller WT. Chest CT features of community-acquired respiratory viral infections in adult inpatients with lower respiratory tract infections. J Thorac Imaging 25: 68$75,2010$.

15. Nabeya D, Kinjo T, Parrott GL, et al. The clinical and phylogenetic investigation for a nosocomial outbreak of respiratory syncytial virus infection in an adult hemato-oncology unit. J Med Virol 89: 1364-1372, 2017.

16. Hirsch HH, Martino R, Ward $\mathrm{KN}$, Boeckh $\mathrm{M}$, Einsele $\mathrm{H}$, Ljungman P. Fourth European Conference on Infections in Leukaemia (ECIL-4): guidelines for diagnosis and treatment of human respiratory syncytial virus, parainfluenza virus, metapneumovirus, rhinovirus, and coronavirus. Clin Infect Dis 56: 258-266, 2013.

17. Dawson KP, Long A, Kennedy J, Mogridge N. The chest radiograph in acute bronchiolitis. J Paediatr Child Health 26: 209-211, 1990.

18. Mansbach JM, Piedra PA, Teach SJ, et al. Prospective multicenter study of viral etiology and hospital length of stay in children with severe bronchiolitis. Arch Pediatr Adolesc Med 166: 700-706, 2012.

19. Falsey AR. Respiratory syncytial virus infection in adults. Semin Respir Crit Care Med 28: 171-181, 2007.

20. Marchiori E, Escuissato DL, Gasparetto TD, Considera DP, Franquet T. "Crazy-paving" patterns on high-resolution CT scans in patients with pulmonary complications after hematopoietic stem cell transplantation. Korean J Radiol 10: 21-24, 2009.

The Internal Medicine is an Open Access journal distributed under the Creative Commons Attribution-NonCommercial-NoDerivatives 4.0 International License. To view the details of this license, please visit (https://creativecommons.org/licenses/ by-nc-nd/4.0/).

(C) 2020 The Japanese Society of Internal Medicine Intern Med 59: 247-252, 2020 